\title{
Hyperbolic structures on wreath products
}

\author{
Sahana H. Balasubramanya \\ Communicated by Alexander Yu. Olshanskii
}

\begin{abstract}
The study of the poset of hyperbolic structures on a group $G$ was initiated by C. Abbott, S. Balasubramanya and D. Osin, [Hyperbolic structures on groups, Algebr. Geom. Topol. 19 (2019), no. 4, 1747-1835, DOI 10.2140/agt.2019.19.1747]. However, this poset is still very far from being understood, and several questions remain unanswered. In this paper, we give a complete description of the poset of hyperbolic structures on the lamplighter groups $\mathbb{Z}_{n}$ wr $\mathbb{Z}$ and obtain some partial results about more general wreath products. As a consequence of this result, we answer two open questions regarding quasiparabolic structures: we give an example of a group $G$ with an uncountable chain of quasiparabolic structures and prove that the lamplighter groups $\mathbb{Z}_{n}$ wr $\mathbb{Z}$ all have finitely many quasi-parabolic structures.
\end{abstract}

\section{Introduction}

It is common in geometric group theory to study groups via their actions on a metric space. The easiest way to do so is to convert the group $G$ itself into a metric space by fixing a generating set $X$ and endowing $G$ with the corresponding word metric $\mathrm{d}_{X}$; the group has a natural cobounded action on the associated Cayley graph $\Gamma(G, X)$. However, not all generating sets are equally good for this purpose. For example, the Cayley graph corresponding to $X=G$ has diameter 1 and is therefore quasi-isometric to a point. This Cayley graph retains no information about the inherent structure of the group. On the other hand, the Cayley graph in the case when $X$ is finite retains maximum information about the group. In joint work with Carolyn Abbott and Denis Osin in [1], the author focused on formalizing this notion. One important notion introduced in that paper is the set of $h y$ perbolic structures on $G$, denoted $\mathscr{H}(G)$, which consists of equivalence classes of (possibly infinite) generating sets of $G$ such that the corresponding Cayley graph is hyperbolic; two generating sets of $G$ are equivalent if the corresponding word metrics on $G$ are bi-Lipschitz equivalent. These equivalence classes can be ordered according to the amount of information the associated Cayley graph retains about the group (see Section 2 for details); this makes $\mathscr{H}(G)$ a poset.

One basic, yet important result about $\mathscr{H}(G)$ follows from Gromov's classification of groups acting on hyperbolic spaces. Using this classification, it is easy to 
see that $\mathscr{H}(G)$ can be expressed as the following disjoint union:

$$
\mathscr{H}(G)=\mathscr{H}_{\mathrm{e}}(G) \sqcup \mathscr{H}_{\ell}(G) \sqcup \mathscr{H}_{\mathrm{qp}}(G) \sqcup \mathscr{H}_{\mathrm{gt}}(G),
$$

where the sets of elliptic, lineal, quasi-parabolic and general type hyperbolic structures on $G$ are denoted $\mathscr{H}_{\mathrm{e}}(G), \mathscr{H}_{\ell}(G), \mathscr{H}_{\mathrm{qp}}(G)$ and $\mathscr{H}_{\mathrm{gt}}(G)$ respectively (see Section 2 for details).

Out of these, lineal and general type actions were well-examined in [1], and several interesting examples and results were obtained. Of special interest were the following results: given any $n \in \mathbb{N}$, there exist (distinct) finitely generated groups $G_{n}$ and $H_{n}$ such that $\left|\mathscr{H}_{\ell}\left(G_{n}\right)\right|=n$ and $\left|\mathscr{H}_{\mathrm{gt}}\left(H_{n}\right)\right|=n$.

However, the understanding of quasi-parabolic structures is far from being complete. It was shown in [1, Proposition 4.27] that $\mathbb{Z}$ wr $\mathbb{Z}$ has an uncountable antichain of quasi-parabolic structures, but little else is known. The authors of [1] consequently posed the following two open questions.

Problem 1.1. Does there exist a group $G$ such that $\mathscr{H}_{\mathrm{qp}}(G)$ is non-empty and finite?

Problem 1.2. Does there exist a group $G$ such that $\mathscr{H}_{\mathrm{qp}}(G)$ contains an uncountable chain?

The answer to both these questions will be obtained as consequences of a general theorem for wreath products $G$ wr $\mathbb{Z}$ that we will prove in this paper (Theorem 1.4 below). In what follows, $\mathbb{S}_{G}$ denotes the poset of proper subgroups of $G$ ordered by containment.

Definition 1.3. For any group $G$, we will refer to the following poset as $\mathscr{B}(G)$ : two (disjoint) incomparable copies of $\mathbb{S}_{G}$, both of which dominate a common element, which in turn dominates one more element (see Figure 1). In other words, $\mathscr{B}(G)$ contains two copies of $\mathbb{S}_{G}$ such that every element in one copy is incomparable to every element in the other copy of $\mathbb{S}_{G}$. Both copies of $\mathbb{S}_{G}$ dominate one common element, and that element dominates one more element.

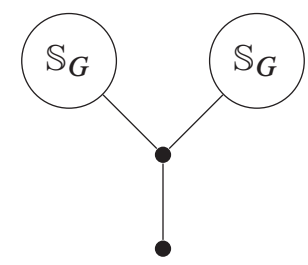

Figure 1. $\mathscr{B}(G) \subset \mathcal{H}(G$ wr $\mathbb{Z})$. 
Theorem 1.4. Let $G$ be a group.

(1) Then $\mathcal{B}(G) \subset \mathscr{H}(G$ wr $\mathbb{Z})$. Specifically, the two copies of $\mathbb{S}_{G}$ correspond to quasi-parabolic structures on $G \mathrm{wr} \mathbb{Z}$. The common element that is dominated by the two copies of $\mathbb{S}_{G}$ corresponds to a lineal structure on $G \mathrm{wr} \mathbb{Z}$. In turn, the lineal structure dominates the trivial structure on $G \mathrm{wr} \mathbb{Z}$.

(2) If $G=\mathbb{Z}_{n}$, then $\mathscr{B}(G)=\mathscr{H}\left(\mathbb{Z}_{n}\right.$ wr $\left.\mathbb{Z}\right)$.

The proof of the above theorem is a combination of the description of quasiparabolic structures obtained (in a different language) in [4], along with elementary (but lengthy) arguments from commutative algebra. Also note that, in general, the equality $B(G)=\mathscr{H}(G \mathrm{wr} \mathbb{Z})$ does not hold. Indeed, this equality does not even hold for every finite group $G$. The case when $G=\mathbb{Z}_{2} \times \mathbb{Z}_{2}$ provides a counterexample, as shown in Example 4.16.

The answer to Problem 1.1 is obtained immediately; the lamplighter groups all have finitely many quasi-parabolic structures. Specifically, the number of quasiparabolic structures on $\mathbb{Z}_{n}$ wr $\mathbb{Z}$ equals twice the number of proper divisors of $n$. The answer to Problem 1.2 is obtained by applying Theorem 1.4 (1) to the group $\mathbb{F}_{2}$ wr $\mathbb{Z}$. Indeed, it is easy to show that the poset of subsets of $\mathbb{N}$ ordered by containment, denoted $\mathbb{P}(\mathbb{N})$, embeds into $\mathbb{S}_{\mathbb{F}_{2}}$. We thus obtain the following corollary.

Corollary 1.5. $\mathscr{H}_{\mathrm{qp}}\left(\mathbb{F}_{2}\right.$ wr $\left.\mathbb{Z}\right)$ contains an isomorphic copy of $\mathbb{P}(\mathbb{N})$. In particular, $\mathscr{H}_{\mathrm{qp}}\left(\mathbb{F}_{2}\right.$ wr $\left.\mathbb{Z}\right)$ contains an uncountable chain.

Please note that it was shown in [1] that the Baumslag-Solitar group BS $(1,2)$ has at least two distinct quasi-parabolic structures, but the exact number was not known at that time. In more recent work, following the completion of this paper, Abbott and Rasmussen use techniques similar to those in this paper to give a complete description of the quasi-parabolic structures on $\mathrm{BS}(1, n)$ in [2].

Although this paper does provide some answers, there are others that remain unanswered. One question relates to the fact that the lamplighter groups (and $\mathrm{BS}(1, n))$ all have an even number of quasi-parabolic structures. We may thus ask if there exists a group $G$ such that $\left|\mathscr{H}_{\mathrm{qp}}(G)\right|$ is odd. More generally, we can also ask whether there exist groups $G_{n}$ such that $\left|\mathscr{H}_{\mathrm{qp}}\left(G_{n}\right)\right|=n$ for every $n \geq 1$. One can also ask which conditions the group $G$ would need to satisfy in order to ensure that $\mathscr{B}(G)=\mathscr{H}(G$ wr $\mathbb{Z})$.

Organization of the paper. The next section will list the required preliminary information. Section 3 focuses on proving the first part of Theorem 1.4, from which Corollary 1.5 will follow immediately. Section 4 discusses the structure of the lamplighter groups and proves the second part of Theorem 1.4 by giving a complete description of $\mathscr{H}\left(\mathbb{Z}_{n}\right.$ wr $\left.\mathbb{Z}\right)$. 


\section{Preliminaries}

Comparing generating sets and group actions. We begin by recalling some standard terminology and definitions. Throughout this paper, all group actions on metric spaces are assumed to be isometric. Given a metric space $S$, we denote by $\mathrm{d}_{S}$ the distance function on $S$ unless another notation is introduced explicitly. Our standard notation for an action of a group $G$ on a metric space $S$ is $G \curvearrowright S$. Given a point $s \in S$ or a subset $X \subseteq S$ and an element $g \in G$, we denote by $g s$ (respectively, $g X$ ) the image of $s$ (respectively, $X$ ) under the action of $g$. Given a group $G \curvearrowright S$ and some $s \in S, G s$ denotes the $G$-orbit of $s$.

Definition 2.1. An action of a group $G$ on a metric space $S$ is said to be

(i) proper if, for every bounded subset $B \subseteq S$, the set $\{g \in G \mid g B \cap B \neq \emptyset\}$ is finite,

(ii) cobounded if there exists a bounded subset $B \subseteq S$ such that $S=\bigcup_{g \in G} g B$,

(iii) geometric if it is proper and cobounded.

Definition 2.2. A map $f: R \rightarrow S$ between two metric spaces $R$ and $S$ is a quasiisometric embedding if there is a constant $C$ such that, for all $x, y \in R$, we have

$$
\frac{1}{C} \mathrm{~d}_{R}(x, y)-C \leq \mathrm{d}_{S}(f(x), f(y)) \leq C \mathrm{~d}_{R}(x, y)+C .
$$

In addition, if $S$ is contained in the $C$-neighborhood of $f(R), f$ is called a quasiisometry. Two metric spaces $R$ and $S$ are quasi-isometric if there is a quasi-isometry $R \rightarrow S$. It is well-known that quasi-isometry of metric spaces is an equivalence relation.

Definition 2.3. Given a group $G$ acting on metric spaces $R, S$, a map $f: R \rightarrow S$ is called $G$-equivariant if $f(g r)=g f(r)$ for every $g \in G$ and every $r \in R$.

We now recall the notion of comparing two generating sets of a group, introduced in [1].

Definition 2.4 ([1, Definition 1.1]). Let $X, Y$ be two generating sets of a group $G$. We say that $X$ is dominated by $Y$, written $X \preceq Y$, if the identity map on $G$ induces a Lipschitz map between metric spaces $\left(G, \mathrm{~d}_{Y}\right) \rightarrow\left(G, \mathrm{~d}_{X}\right)$. This is equivalent to the requirement that $\sup _{y \in Y}|y|_{X}<\infty$, where $|\cdot|_{X}=\mathrm{d}_{X}(1, \cdot)$ denotes the word length with respect to $X$. It is easy to see that $\preceq$ is a preorder on the set of generating sets of $G$, and therefore it induces an equivalence relation in the standard way,

$$
X \sim Y \Longleftrightarrow X \preceq Y \text { and } Y \preceq X
$$


This is equivalent to the condition that the Cayley graphs $\Gamma(G, X)$ and $\Gamma(G, Y)$ are $G$-equivariantly quasi-isometric. We denote by $[X]$ the equivalence class of a generating set $X$, and by $\mathscr{G}(G)$ the set of all equivalence classes of generating sets of $G$. The preorder $\preceq$ induces an order relation $\preccurlyeq$ on $\mathscr{G}(G)$ by the rule

$$
[X] \preccurlyeq[Y] \Longleftrightarrow X \preceq Y .
$$

For example, all finite generating sets of a finitely generated group are equivalent, and the corresponding equivalence class is the largest element of $\mathscr{G}(G)$. For every group $G,[G]$ is the smallest element of $\mathcal{G}(G)$. Note also that our order is "inclusion reversing": if $X$ and $Y$ are generating sets of $G$ such that $X \subseteq Y$, then $Y \preceq X$.

To define a hyperbolic structure on a group, we first recall the definition of a hyperbolic space. In this paper, we employ the definition of hyperbolicity via the Rips condition.

Definition 2.5. A metric space $S$ is called $\delta$-hyperbolic if it is geodesic and, for any geodesic triangle $\Delta$ in $S$, each side of $\Delta$ is contained in the union of the closed $\delta$-neighborhoods of the other two sides.

Definition 2.6 ([1, Definition 1.2]). A hyperbolic structure on $G$ is an equivalence class $[X] \in \mathscr{G}(G)$ such that $\Gamma(G, X)$ is hyperbolic. Since hyperbolicity of a space is a quasi-isometry invariant, this definition is independent of the choice of a particular representative in the equivalence class $[X]$. We denote the set of hyperbolic structures by $\mathscr{H}(G)$ and endow it with the order induced from $\mathscr{E}(G)$.

Alternatively, $\mathscr{H}(G)$ can be described in terms of general cobounded actions of the group $G$ on hyperbolic spaces by utilizing the Svarc-Milnor map, which allows us to interchangeably work with generating sets or cobounded group actions. However, knowledge of this notion is not essential in this paper, and so we refer the reader to [1, Section 3] for details.

General classification of hyperbolic structures. We now recall some standard facts about groups acting on hyperbolic spaces. For details, the reader is referred to [5]. Given a hyperbolic space $S$, we denote by $\partial S$ its Gromov boundary. In general, $S$ is not assumed to be proper. Thus the boundary is defined as the set of equivalence classes of sequences convergent at infinity. Given a group $G$ acting on a hyperbolic space $S$, by $\Lambda(G)$ we denote the set of limit points of $G$ on $\partial S$. That is,

$$
\Lambda(G)=\partial S \cap \overline{G S},
$$

where $\overline{G s}$ denotes the closure of a $G$-orbit in $S \cup \partial S$; it is easy to show that 
this definition is independent of the choice of $s \in S$. The action of $G$ is called elementary if $|\Lambda(G)| \leq 2$ and non-elementary otherwise. The action of $G$ on $S$ naturally extends to a continuous action of $G$ on $\partial S$.

Definition 2.7. Given an action of a group $G$ on a hyperbolic space $S$, an element $g \in G$ is called

(i) elliptic if $\langle g\rangle$ has bounded orbits,

(ii) loxodromic if the map $n \mapsto g^{n} s, n \in \mathbb{Z}$, is a quasi-isometric embedding for some (equivalently any) $s \in S$,

(iii) parabolic otherwise.

Every loxodromic element $g \in G$ has exactly two fixed points $g^{ \pm \infty}$ on $\partial S$, where $g^{+\infty}$ (respectively, $g^{-\infty}$ ) is the limit of the sequence $\left(g^{n} s\right)_{n \in \mathbb{N}}$ (respectively, $\left.\left(g^{-n} s\right)_{n \in \mathbb{N}}\right)$ for any fixed $s \in S$. We clearly have $\Lambda(\langle g\rangle)=\left\{g^{ \pm \infty}\right\}$.

The following theorem summarizes the standard classification of groups acting on hyperbolic spaces due to Gromov [5, Section 8.2] (see also [6] for complete proofs in a more general context) and some results from [4, Propositions 3.1 and 3.2].

Theorem 2.8. Let $G$ be a group acting on a hyperbolic space $S$. Then exactly one of the following conditions holds.

(1) $|\Lambda(G)|=0$. Equivalently, $G$ has bounded orbits. In this case, the action of $G$ is called elliptic.

(2) $|\Lambda(G)|=1$. In this case, the action of $G$ is called parabolic. A parabolic action cannot be cobounded.

(3) $|\Lambda(G)|=2$. Equivalently, $G$ contains a loxodromic element and any two loxodromic elements have the same limit points on $\partial S$. In this case, the action of $G$ is called lineal.

(4) $|\Lambda(G)|=\infty$. Then $G$ always contains loxodromic elements. In turn, this case breaks into two subcases.

(a) G fixes a point of $\partial S$. Equivalently, any two loxodromic elements of $G$ have a common limit point on the boundary. In this case, the action of $G$ is called quasi-parabolic or focal.

(b) $G$ does not fix any point of $\partial S$. In this case, the action of $G$ is said to be of general type.

As a consequence of the above theorem, we proved the following classification of hyperbolic structures in [1]. 
Theorem 2.9 ([1, Theorem 4.6]). For every group $G$,

$$
\mathscr{H}(G)=\mathscr{H}_{\mathrm{e}}(G) \sqcup \mathscr{H}_{\ell}(G) \sqcup \mathscr{H}_{\mathrm{qp}}(G) \sqcup \mathscr{H}_{\mathrm{gt}}(G),
$$

where the sets of elliptic, lineal, quasi-parabolic and general type hyperbolic structures on $G$ are denoted by $\mathscr{H}_{\mathrm{e}}(G), \mathscr{H}_{\ell}(G), \mathscr{H}_{\mathrm{qp}}(G)$ and $\mathscr{H}_{\mathrm{gt}}(G)$ respectively.

The Busemann pseudocharacter. A map $q: G \rightarrow \mathbb{R}$ is a quasi-character if there exists a constant $D$ such that $|q(g h)-q(g)-q(h)| \leq D$ for all $g, h \in G$. We say that $q$ has defect at most $D$. If, in addition, the restriction of $q$ to every cyclic subgroup of $G$ is a homomorphism, $q$ is called a pseudocharacter. Every quasicharacter $q$ gives rise to a pseudocharacter $p$ defined by

$$
p(g)=\lim _{n \rightarrow \infty} \frac{q\left(g^{n}\right)}{n}
$$

(the limit always exists); $p$ is called the homogenization of $q$. It is straightforward to check that $|p(g)-q(g)| \leq D$ for all $g \in G$ if $q$ has defect at most $D$.

Given any action of a group on a hyperbolic space fixing a point on the boundary, one can associate the so-called Busemann pseudocharacter. We briefly recall the construction and necessary properties here and refer to [5, Section 7.5.D] and $[8$, Section 4.1] for more details.

Definition 2.10. Let $G$ be a group acting on a hyperbolic space $S$ and fixing a point $\xi \in \partial S$. Fix any $s \in S$, and let $\mathbf{x}=\left(x_{i}\right)$ be any sequence of points of $S$ converging to $\xi$. Then the function $q_{\mathbf{x}}: G \rightarrow \mathbb{R}$ defined by

$$
q_{\mathbf{x}}(g)=\limsup _{n \rightarrow \infty}\left(\mathrm{d}_{S}\left(g s, x_{n}\right)-\mathrm{d}_{S}\left(s, x_{n}\right)\right)
$$

is a quasi-character. Its homogenization $p_{\mathbf{x}}$ is called the Busemann pseudocharacter. It is known that this definition is independent of the choice of $\mathbf{x}$ (see [8, Lemma 4.6]), and thus we can drop the subscript in $p_{\mathbf{x}}$. It is straightforward to verify that $g \in G$ acts loxodromically on $S$ if and only if $p(g) \neq 0$; in particular, $p$ is non-zero whenever $G \curvearrowright S$ is quasi-parabolic.

Definition 2.11. A quasi-parabolic action of a group on a hyperbolic space is called regular (or regular focal) if the associated Busemann pseudocharacter is a homomorphism.

\section{Quasi-parabolic structures on $G$ wr $\mathbb{Z}$}

To prove the first part of Theorem 1.4, we will first introduce some important notions related to (restricted) wreath products. The following visualization of some 
elements from $G$ wr $\mathbb{Z}$ will be very useful in making several arguments in this section: let $A=\bigoplus_{\mathbb{Z}} G$ denote the base of the wreath product. We naturally think of elements of $A$ as functions from $\mathbb{Z}$ to $G$ with finite support, i.e., only finitely many values in the image can be distinct from $e \in G$. This is a group under coordinate-wise group operation, which we will denote using multiplicative notation. Let $\mathbb{Z}=\langle t\rangle$. Then the action of $t$ on $A$ is by conjugation and $G$ wr $\mathbb{Z}=A \rtimes\langle t\rangle$.

The following notions are taken from [4]. These will serve as important tools in proving Theorem 1.4.

Definition 3.1 ([4, Section 4]). Let $(A, *)$ be a group and $Q$ a subset of $A$, and let $\alpha$ be an automorphism of $A$. We say that the action of $\alpha$ is (strictly) confining $A$ into $Q$ if it satisfies the following conditions:

(a) $\alpha(Q)$ is (strictly) contained in $Q$;

(b) $A=\bigcup_{n \geq 0} \alpha^{-n}(Q)$;

(c) $\alpha^{n_{0}}(Q * Q) \subset Q$ for some non-negative integer $n_{0}$.

Proposition 3.2 ([4, Proposition 4.6]). Let $A$ be a group, and let $\alpha$ be an automorphism of $A$ which confines $A$ into some subset $Q \subset A$. Let $S=\left\{Q, \alpha^{ \pm 1}\right\}$. Then the group $G=A \rtimes\langle\alpha\rangle$ is Gromov hyperbolic with respect to the left-invariant word metric associated to the generating set $S$. If the containment $\alpha(Q) \subset Q$ is strict, then it is (regular) focal.

Although the conclusion of the proposition in [4] does not state that we get a regular action, it is not too hard to see that this is indeed the case. We include a brief outline (provided by Y. de Cornulier) here. The proof of [4, Proposition 4.6] shows that $\alpha$ is a loxodromic element with respect to the action of $G$ of $\Gamma(G, S)$ and that this action fixes one of the end points $\left\{\alpha^{ \pm \infty}\right\} \in \partial X$. It can be shown using the techniques developed in [4, Section 4] that $q_{\mathbf{x}}\left(w \alpha^{k}\right)-k$ is bounded independently of $w \in A, k \in \mathbb{Z}$. From this, it follows that the associated Buseman pseudocharacter is equal to the map $w \alpha^{k} \mapsto k$.

Remark 3.3. If the action is confining but not strictly confining, i.e., $\alpha(Q)=Q$, then the above theorem still holds with the difference that $\left(G, d_{S}\right)$ is quasi-isometric to a line; the resulting action is thus lineal. Indeed, if $\alpha(Q)=Q$, then $A=Q$ is bounded. Thus the above theorem implies that when the action is strictly confining, then $[S] \in \mathscr{H}_{\mathrm{qp}}(G)$.

To prove Theorem 1.4(1), we will describe subsets $Q \subset A$ associated to each proper subgroup $H<G$ such that the action of either $t$ or $t^{-1}$ is strictly confining $A$ into $Q$. Note that, in the case of the wreath product, the action is by conjugation and that we read our elements from left to right. Our standard notation will 
be $t . b=t b t^{-1}, t^{-1} \cdot b=t^{-1} b t$, unless otherwise specified. We now restate the conditions of Definition 3.1 for the specific case of the wreath product. For this, we will need the following definition. Recall that $A$ is the set of functions from $\mathbb{Z}$ to $G$ with finite support.

Definition 3.4 (Multiplying a function by $t$ or $t^{-1}$ ). Let $\mathbb{Z}=\langle t\rangle$, and let $f \in A$ be a function. Then $t . f \in A$ is the function given by

$$
\text { t. } f(i+1)=f(i) \text { for all } i \in \mathbb{Z} .
$$

The function $t^{-1} . f$ is defined similarly. Observe that, since $f$ has finite support, so do $t . f$ and $t^{-1} . f$. Indeed, $t . f$ and $t^{-1} . f$ can be thought of as the "right shift" and "left shift" of $f$ respectively, while leaving the marker unchanged.

Definition 3.5. Let $\mathbb{Z}=\langle t\rangle$ and $Q \subset A$. The action of $t$ is (strictly) confining $A$ into $Q$ if

(a) t.Q is (strictly) contained in $Q$,

(b) for every $f \in A$, there exists $n \geq 0$ such that $t^{n} . f \in Q$,

(c) there exists a constant integer $n_{0} \geq 0$ such that $t^{n_{0}} .(Q Q) \subset Q$.

We will now prove the first part of Theorem 1.4 in a series of smaller results. We start by showing how to create two quasi-parabolic structures given a proper subgroup of $G$. Recall that $\mathbb{S}_{G}$ is the poset of proper subgroups of $G$, ordered by containment.

Lemma 3.6. Let $H \in \mathbb{S}_{G}$. Then the action of $t$ is strictly confining $A$ into the set $Q_{H} \subset A$ given by

$$
Q_{H}=\{f \in A \mid f(-i) \in H \text { for all } i>0\} .
$$

Proof. We prove the lemma by verifying the conditions of Definition 3.5. Let $f \in Q_{H}$. Then $t$. $f$ satisfies $t . f(-i)=f(-i-1) \in H$ for all $i \geq 1$. Thus we have $t . f \in Q_{H}$. To see that the containment of $t . Q_{H}$ into $Q_{H}$ is strict, observe that, since $H$ is a proper subgroup of $G$, there exists $g \in G \backslash H$. Define a function $p \in A$ by $p(0)=g$ and identity everywhere else. Then $p \notin t . Q_{H}$. Indeed, if $p \in t . Q_{H}$, then we must have a function $p^{\prime} \in Q_{H}$ such that $p^{\prime}(-1) \notin H$, which is impossible. This proves condition (a) of Definition 3.5.

Given any $f \in A, f$ has finite support. Thus there exists a minimal negative integer $-n$ in the support of $f$. But then $t^{n} . f \in Q_{H}$ since $\left(t^{n} \cdot f\right)(-i)=e$ for all $i \geq 1$. Thus condition (b) of Definition 3.5 holds. To see that condition (c) holds, observe that $\left(Q_{H}\right)\left(Q_{H}\right) \subset Q_{H}$ since $H$ is a subgroup and the functions are combined component-wise. So condition (c) holds with $n_{0}=0$. 
Remark 3.7. The above lemma would be true even if $H$ were a proper submonoid of $G$. The structures arising from submonoids are potentially different from the ones arising from subgroups. The easiest example to consider is when $M=\{0,2,4,6, \ldots\}$ is the submonoid of $\mathbb{Z}$ and $G=\mathbb{Z}$ wr $\mathbb{Z}$.

Similar to the above lemma, we can prove the following.

Lemma 3.8. Let $H \in \mathbb{S}_{G}$. Then the action of $t^{-1}$ is strictly confining $A$ into the set $Q_{H}^{\prime} \subset$ A given by

$$
Q_{H}^{\prime}=\{f \in A \mid f(i) \in H \text { for all } i>0\} .
$$

Note that $Q_{H}, Q_{H}^{\prime}$ are non-empty since the function that takes identity everywhere is in both sets. Thus each $H \in \mathbb{S}_{G}$ defines two quasi-parabolic structures on $G$ wr $\mathbb{Z}$ given by the equivalence classes of the generating sets $S_{H}=\left\{Q_{H}, t^{ \pm 1}\right\}$ and $S_{H}^{\prime}=\left\{Q_{H}^{\prime}, t^{ \pm 1}\right\}$. We will fix this notation for the rest of the paper.

Lemma 3.9. The maps $\psi, \psi^{\prime}: \mathbb{S}_{G} \rightarrow \mathscr{H}_{\mathrm{qp}}(G)$ given by $H \mapsto\left[S_{H}\right]$ and $H \mapsto\left[S_{H}^{\prime}\right]$ respectively are order reversing.

Proof. We will prove the lemma for the map $\psi$; the arguments for the map $\psi^{\prime}$ are symmetric. Let $H, K \in \mathbb{S}_{G}$ such that $H \leq K$. Let $f \in Q_{H}$. Then it is easy to see that $f \in Q_{K}$ also. Thus $\sup _{x \in S_{H}}|x|_{S_{K}}=1$, and so $\left[S_{K}\right] \preccurlyeq\left[S_{H}\right]$. Thus $\psi$ is order reversing.

Lemma 3.10. The maps $\psi, \psi^{\prime}$ defined in Lemma 3.9 are injective.

Proof. We will prove the lemma for the map $\psi$; the arguments for the map $\psi^{\prime}$ are symmetric. Let $H, K \in \mathbb{S}_{G}$ such that $H \neq K$. Without loss of generality, we may assume that $H \not \leq K$. Choose an element $h \in H \backslash K$. Define the following functions in $A$ for each $i \geq 1$ :

$$
p_{i}(-i)=h \quad \text { and } \quad p_{i} \text { is identity everywhere else. }
$$

Then it is easy to check that $\sup _{i}\left|p_{i}\right| S_{H}=1$, but $\sup _{i}\left|p_{i}\right|_{S_{K}}=+\infty$. Thus we have $\left[S_{H}\right] \neq\left[S_{K}\right]$.

Lemma 3.11. Let $H, K \in \mathbb{S}_{G}$. Then the structures $\left[S_{H}\right]$, $\left[S_{K}^{\prime}\right]$ are incomparable.

Proof. Since $H<G$, there exists an element $g \in G \backslash H$. It is easy to verify that the functions $f_{i} \in A$ for $i \geq 1$, given by

$$
f_{i}(-i)=g \text { and } f_{i} \text { is identity everywhere else }
$$

satisfy $\sup _{i}\left|f_{i}\right|_{S_{K}^{\prime}}=1$, while $\sup _{i}\left|f_{i}\right|_{S_{H}}=+\infty$. 
Similarly, we can find an element $g^{\prime} \in G \backslash K$. Then the functions $p_{i}, i \geq 1$ given by

$$
p_{i}(i)=g^{\prime} \quad \text { and } \quad p_{i} \text { is identity everywhere else }
$$

satisfy the condition that $\sup _{i}\left|p_{i}\right|_{S_{H}}=1$, but $\sup _{i}\left|p_{i}\right|_{S_{K}^{\prime}}=+\infty$. Thus $\left[S_{H}\right]$ and $\left[S_{K}^{\prime}\right]$ are incomparable.

Proof of Theorem 1.4 (1). By Lemmas 3.9 and 3.10, the maps $\psi, \psi^{\prime}$ create two copies of $\mathbb{S}_{G}$ in $\mathscr{H}_{\mathrm{qp}}(G \mathrm{wr} \mathbb{Z})$. That these copies are incomparable follows from Lemma 3.11.

Consider the generating set $L=\left\{A, t^{ \pm 1}\right\}$ for $G$ wr $\mathbb{Z}$. It is easy to see that the Cayley graph of $G$ wr $\mathbb{Z}$ with respect to the generating set $L$ is quasi-isometric to a line and hence hyperbolic. Further, $t$ acts as a loxodromic element in this Cayley graph, and thus the action is lineal. Since $Q_{H}, Q_{H}^{\prime} \subset A$ for every $H<G$, it follows that $[L] \preccurlyeq\left[S_{H}\right]$ and $[L] \preccurlyeq\left[S_{H}^{\prime}\right]$ for every $H<G$. Thus the two copies of $\mathbb{S}_{G}$ dominate a common lineal structure, which in turn obviously dominates the trivial structure. This results in an embedding of the entire poset from Figure 1 into $\mathscr{H}(G$ wr $\mathbb{Z})$.

Corollary 3.12. There exists a finitely generated group $W$ such that $\mathscr{H}_{\mathrm{qp}}(W)$ contains a chain of cardinality continuum.

Proof. Let $\mathbb{F}_{\infty}=\left\langle a_{1}, a_{2}, a_{3}, a_{4}, \ldots\right\rangle$ be the free group on countably infinitely many generators. For each subset $I \subseteq \mathbb{N}$, set $H_{I}=\left\langle a_{2 i} \mid i \in I\right\rangle$. Then $H_{I}<\mathbb{F}_{\infty}$, and we have the following properties.

(1) If $I \neq J \subset \mathbb{N}$, then $H_{I} \neq H_{J}$ (since the groups are free).

(2) If $I \subset J$, then $H_{I}<H_{J}$.

This creates an embedding of $\mathbb{P}(\mathbb{N})$ into $\mathbb{S}_{\mathbb{F}_{\infty}}$. Set $W=\mathbb{F}_{2}$ wr $\mathbb{Z}$, where $\mathbb{F}_{2}$ is the free group on 2 generators. Since $\mathbb{F}_{\infty}<\mathbb{F}_{2}$, we get the sequence of embeddings

$$
\mathbb{P}(\mathbb{N}) \hookrightarrow \mathbb{S}_{\mathbb{F}_{\infty}} \hookrightarrow \mathbb{S}_{\mathbb{F}_{2}} \hookrightarrow \mathscr{H}_{\mathrm{qp}}(W) .
$$

Since $\mathbb{P}(\mathbb{N})$ contains chains (and antichains) of cardinality continuum, it follows that so does $\mathscr{H}_{\mathrm{qp}}(W)$.

Remark 3.13. Let $G$ be a countable group and $H<G$. Let $H_{j}$ be a copy of $H$ for each $j<0$, and let $G_{j}$ be a copy of $G$ for each $j \geq 0$. Then it is easy to see that $G \mathrm{wr} \mathbb{Z}$ is an ascending HNN-extension of $\prod_{j<0} H_{j} \times \prod_{j \geq 0} G_{j}$.

Let $T_{H}$ be the associated Bass-Serre tree. Since the extension is ascending, the group $G$ wr $\mathbb{Z}$ fixes an end of $T_{H}$, and hence the action of the group on $T_{H}$ is quasi-parabolic. Let $S_{H}$ be as defined after Lemma 3.8. It is also easy to see that 
the action of $G \mathrm{wr} \mathbb{Z}$ on $T_{H}$ yields the quasi-parabolic structure $\left[S_{H}\right]$ on $G$ wr $\mathbb{Z}$ under the Svarc-Milnor map (see [1, Definition 3.13] for details). We can thus conclude that the structure $\left[S_{H}\right]$ corresponds to a Cayley graph of $G$ wr $\mathbb{Z}$ which is a quasi-tree.

In particular, since $\mathbb{F}_{2}$ is countable, we conclude that all the quasi-parabolic structures in the antichain and chain constructed in Corollary 3.12 correspond to Cayley graphs that are quasi-trees.

\section{Quasi-parabolic structures on the lamplighter groups}

The goal of this section is to prove Theorem 1.4 (2). Our approach is to first show that all quasi-parabolic structures of the lamplighter groups are regular, and derive a characterization of quasi-parabolic structures on these groups using techniques developed in [4].

We begin by discussing the structure of the lamplighter groups. Denoted $L_{n}$, $n \geq 2$, the lamplighter groups are given by the presentation

$$
\left.\langle a, t|\left[a^{t^{i}}, a^{t^{j}}\right]=e \text { for all } i, j \in \mathbb{Z}, a^{n}=e\right\rangle,
$$

where $x^{y}=y x y^{-1}$.

Equivalently, this group is the (restricted) wreath product $\mathbb{Z}_{n}$ wr $\mathbb{Z}$ or the semidirect product $\left(\bigoplus_{\mathbb{Z}} \mathbb{Z}_{n}\right) \rtimes \mathbb{Z}$. The "lamplighter" picture of elements of this group is as follows. Take a bi-infinite road of light bulbs placed at integer points. Each bulb has $n$ possible states corresponding to the elements of $\mathbb{Z}_{n}$, given by the powers of $a$. There is also a lamplighter, indicating the particular bulb under consideration. The action of the group on this picture is that $t$ (the generator of $\mathbb{Z}$ ) moves the lamplighter one position to the right, and powers of $a$ change the state of the current bulb under consideration. Thus each element of $L_{n}$ can be interpreted as a configuration of a finite collection of lit lamps in some allowable states, leaving the lamplighter at a fixed integer. The null configuration, denoted $\overline{0}$, is the element where all the lamps are off and the lamplighter stands at index 0 . Note that we read the elements of the group from left to right applied to the null configuration. We will fix the above presentation for the remainder of this paper.

Let $A=\bigoplus_{\mathbb{Z}} \mathbb{Z}_{n}$. The visualization of $A$ in $L_{n}$ is that of elements consisting of a finite number of illuminated lamps in some allowable states, while the lamplighter stands at index 0 . In this section, we will use the description of elements of $L_{n}$ in terms of group rings in order to make several arguments. Let $R=\mathbb{Z}_{n}[\mathbb{Z}]$ be the group ring of Laurent polynomials in $t$ with coefficients in $\mathbb{Z}_{n}$. For $b \in A$, $k \in\{0,1, \ldots, n-1\}$ and $m \in \mathbb{Z}$, define

$$
k t^{m} \cdot b=\left(b^{k}\right)^{t^{m}}=t^{m} b^{k} t^{-m} .
$$


Lemma 4.1. $A$ is a free (left) $R$-module, where the module multiplication is defined by extending $(*)$ canonically to formal sums in $R$. Further, every $b \in A$ can be represented as $p(t)$.a, where $p(t) \in R$. Thus $A$ is isomorphic to $R$ as $R$-modules.

Proof. Observe that $A$ is an abelian group under component-wise group operation. Further, the module multiplication is well defined and sends elements of $A$ to $A$ since the lamplighter remains at index 0 . Let $x, y \in R$ and $b, c \in A$. Clearly, by definition, we have $(x+y) \cdot b=(x \cdot b)(y \cdot b)$. Further, since shifting and combining configurations of lamps (with lamplighter at zero) is equal to combining the configurations and then shifting, we also have $x .(b c)=(x . b)(x . c)$. Lastly, it is easy to see that the set $\{a\} \subset A$ is a basis for $A$ as an $R$-module.

Now let $b \in A$. Then $b=\left(t^{i_{1}} a^{k_{1}} t^{-i_{1}}\right)\left(t^{i_{2}} a^{k_{2}} t^{-i_{2}}\right) \ldots\left(t^{i_{l}} a^{k_{l}} t^{-i_{l}}\right)$ for some indices $\left\{i_{j}, j=1,2, \ldots, l\right\} \subset \mathbb{Z}$ and elements $k_{j} \in\{1,2, \ldots, n-1\}$. Set

$$
p_{b}(t)=k_{1} t^{i_{1}}+k_{2} t^{i_{2}}+\cdots+k_{l} t^{i_{l}} .
$$

Then $p_{b}(t) \in R$ and $p_{b}(t) \cdot a=\left(t^{i_{1}} a^{k_{1}} t^{-i_{1}}\right)\left(t^{i_{2}} a^{k_{2}} t^{-i_{2}}\right) \ldots\left(t^{i_{l}} a^{k_{l}} t^{-i_{l}}\right)=b$.

Since every ring is a module over itself, $R$ is also an $R$-module. We define a map $f: A \rightarrow R$ by the rule $f(b)=p_{b}(t)$. It is easy to verify that this map is a bijective $R$-module homomorphism.

Lemma 4.2. Every element $g \in L_{n}$ can be represented as $t^{m}(p(t) . a)$ for some $m \in \mathbb{Z}, p(t) \in R$.

Proof. Every element $g \in L_{n}$ can be represented as $g=t^{m} b$ for some $m \in \mathbb{Z}$ and some $b \in A$. The result now follows from Lemma 4.1.

Remark 4.3. The lamplighter groups $L_{n}$ are amenable. It thus follows from [4, Corollary 3.9] that all quasi-parabolic actions of the lamplighter groups are regular. Indeed, the Busemann pseudocharacter associated to any quasi-parabolic action of $L_{n}$ is the standard epimorphism to $\mathbb{Z}$.

We will now derive the following characterization of quasi-parabolic structures on $L_{n}=\mathbb{Z}_{n}$ wr $\mathbb{Z}$, which is similar to [4, Theorem 4.1].

Theorem 4.4. $[X] \in \mathscr{H}_{\mathrm{qp}}\left(L_{n}\right)$ if and only if there exists $Q \subset A$ such that the action of $t$ or $t^{-1}$ is strictly confining $Q$ into $A$ and $[X]=\left[\left\{Q, t^{ \pm 1}\right\}\right]$.

In general, the above characterization may not hold for a generic $G=H$ wr $\mathbb{Z}$. Indeed, a primary obstruction would be if $\frac{G}{[G, G]}$ contained a free abelian group of rank greater than 1 . However, this obstruction is not present when $H=\mathbb{Z}_{n}$. To prove the theorem, we first prove the following lemma. 
Lemma 4.5 (cf. [4, Proposition 4.5]). Let $X$ be a generating set of $L_{n}$ such that $\Gamma\left(L_{n}, X\right)$ is hyperbolic and $L_{n} \curvearrowright \Gamma\left(L_{n}, X\right)$ is quasi-parabolic. Let $p$ be the associated Buseman character to this action, and let $B(e, r)$ denote the ball of radius $r$ around the identity $e$ in $\Gamma\left(L_{n}, X\right)$. Then $t \notin \operatorname{ker}(p)$, and there exists an $r_{0}>0$ satisfying: for all $r>0$, there exists an $n_{0}$ such that, for $n \geq n_{0}$,

$$
t^{n}(B(e, r) \cap A) t^{-n} \subset B\left(e, r_{0}\right) \cap A .
$$

In particular, $t$ is confining $A$ into $Q=B\left(e, r_{0}\right) \cap A$.

Proof. By Remark 4.3, the action is regular quasi-parabolic, and thus $p$ is a character. Assume by contradiction that $p(t)=0$. We will show that $p(g)=0$ for every $g \in L_{n}$, which will contradict the assumption that the action is quasi-parabolic.

Observe that every $b \in A$ has finite order, and thus $p(b)=0$ for all $b \in A$. Let $g \in L_{n}$ be any element. Then $g=b t^{k}$ for some $b \in A, k \in \mathbb{Z}$. Since $p$ is a character, we have

$$
p(g)=p\left(b t^{k}\right)=p(b)+p\left(t^{k}\right)=0+k p(t)=0 .
$$

Thus $t \notin \operatorname{ker}(p)$, and so $t$ is a loxodromic element. Consequently, the global fixed point of the boundary is either the attracting or repelling point of $t$. Up to changing $t$ with $t^{-1}$, we may assume without loss of generality that the global fixed point is the repelling point, which we denote $t^{-\infty}$. In particular, connecting consecutive points of the sequence $1, t^{-1}, t^{-2}, t^{-3}, \ldots$ by geodesics defines a quasigeodesic path to $t^{-\infty}$. Therefore, so does the sequence $g, g t^{-1}, g t^{-2}, g t^{-3}, \ldots$ for any $g \in L_{n}$ by taking an image of the entire quasi-geodesic under $g$. Observe that if the sequence $1, t^{-1}, t^{-2}, t^{-3}, \ldots$ defines a $(\lambda, \epsilon)$ quasi-geodesic, then so does $g, g t^{-1}, g t^{-2}, g t^{-3}, \ldots$ since the action is isometric.

It follows from the stability of finite length quasi-geodesics [3, III. Theorem 1.7] and the fact that quasi-geodesic triangles are thin in a hyperbolic space that there exists a constant $r_{0}$ such that any two $(\lambda, \epsilon)$ quasi-geodesic rays with the same end point at infinity are eventually $r_{0}$ close to each other. Note that the constant $r_{0}$ depends on the hyperbolicity constant of $\Gamma\left(L_{n}, X\right), \lambda$ and $\epsilon$.

In particular, it follows that, for $b \in A$, we have that $d_{X}\left(t^{-n}, b t^{-n}\right) \leq r_{0}$ for all $n$ larger than some $n_{0}$, where $n_{0}$ depends only on $d_{X}(1, b)$. The conclusion that $t^{n}(B(e, r) \cap A) t^{-n} \subset B\left(e, r_{0}\right) \cap A$ for all $n \geq n_{0}$ obviously follows, where $n_{0}$ depends on $r$. Conditions (b) and (c) of Definition 3.5 follow from this. If $d_{X}(1, b) \leq r_{0}$, then $n_{0}=0$, and so $d_{X}\left(t^{-1}, b t^{-1}\right) \leq r_{0}$ also. Thus condition (a) of Definition 3.5 also holds, and therefore so does the claim that $t$ is confining $A$ into $B\left(e, r_{0}\right) \cap A$.

Proof of Theorem 4.4. The converse implication follows from [4, Proposition 4.6] and Remark 4.3. Indeed, any subset $Q \subset A$ such that the action of $t$ or $t^{-1}$ is 
strictly confining $A$ into $Q$ defines the (regular) quasi-parabolic structure given by $\left[\left\{Q, t^{ \pm 1}\right\}\right]$.

The forward implication follows from Lemma 4.5. Given $[X] \in \mathscr{H}_{\mathrm{qp}}\left(L_{n}\right)$, we consider the action of $L_{n}$ on $\Gamma\left(L_{n}, X\right)$. This gives us a subset $Q=B\left(e, r_{0}\right) \cap A$ of $A$ such that $t$ confines $A$ into $Q$. Set $S=\left\{Q, t^{ \pm 1}\right\}$. We will show that the identity map $i:\left(A \rtimes\langle t\rangle, d_{S}\right) \rightarrow\left(A \rtimes\langle t\rangle, d_{X}\right)$ is a quasi-isometry. This map is obviously surjective. Since $S$ is bounded in $d_{X}$, the map $i$ is Lipschitz. It thus suffices to prove that if a set is bounded in $d_{X}$, then it is bounded in $d_{S}$. In restriction to $A$, the result follows from Lemma 4.5.

Further, let $g=b t^{k}$ for some $b \in A, k \in \mathbb{Z}$. Since $p$ is a character and $p(t) \neq 0$, we get that $p\left(b t^{k}\right)=p(b)+k p(t)=k p(t)$. From the definition of the Buseman character, we can see that $q(g) \leq d_{X}(1, g)$. Since $|p(g)-q(g)| \leq D$, we get that $-d_{X}(1, g)-D \leq p(g) \leq d_{X}(1, g)+D$, and so

$$
-d_{X}(1, g)-D \leq k p(t) \leq d_{X}(1, g)+D .
$$

Thus $p$ maps bounded sets of $\left(L_{n}, X\right)$ to bounded subsets of $\mathbb{R}$. Consequently, if $g \in L_{n}$ such that $d_{X}(1, g) \leq r$, then $g=b t^{k}$, where $b \in A$ and $k \in \mathbb{Z}$, and there exists a uniform bound $M$ on $k$ that depends on $r$. It follows that $d_{S}(1, g)$ can then be bounded in terms of $r, r_{0}$ and $M$, and so $[X]=[S]$. If $t Q=Q$, then it follows from Remark 3.3 that $[S]$ is a lineal structure, which is impossible. Thus $t Q$ is strictly contained in $Q$.

Since $A \cong R$ when $G=\mathbb{Z}_{n}$, we may prove the results in this section in terms of the elements of $R$ (which are Laurent polynomials) instead of the functions from $A$. This simplifies several notions; for example, the multiplication of elements of $R$ by $t, t^{-1}$ is akin to the multiplication of Laurent polynomials, and so we will henceforth drop the "." in the notation $t$. $f$ and simply write $t f$. In view of Theorem 4.4, we will show that, given any subset $Q \subset A$ such that the action of either $t$ or $t^{-1}$ is strictly confining $A$ into $Q$, there exists a subgroup $H<G$ such that $\left[\left\{Q, t^{ \pm 1}\right\}\right]$ is equivalent to the structure $\left[S_{H}\right]$ or $\left[S_{H}^{\prime}\right]$ respectively. To show this, we will first prove the following proposition.

Proposition 4.6. Let $Q \subset R$, and let the action of t be strictly confining $R$ into $Q$. Let $S=\left\{Q, t^{ \pm 1}\right\}$. Then there exists a subgroup $H<G$ such that $[S]=\left[S_{H}\right]$.

Indeed, if this proposition is proved, then, by symmetric arguments, it will follow that if the action of $t^{-1}$ is strictly confining $R$ into $Q$, then there exists a subgroup $H<G$ such that $[S]=\left[S_{H}^{\prime}\right]$. This will imply that the set of quasi-parabolic structures on $L_{n}$ corresponds precisely to the two copies of $\mathbb{S}_{G}$ in $\mathcal{B}(G)$.

For the sake of convenience, we will let $B_{+}$and $B_{-}$denote the subsets of $R$ that yield the quasi-parabolic structures corresponding to the trivial subgroup $\{e\}$ 
of $\mathbb{Z}_{n}$, i.e., $B_{+}$denotes the set of all elements from $R$ with only non-negative powers of $t$, and $B_{-}$denotes the set of all elements from $R$ with only non-positive powers of $t$. We also denote by $\widetilde{Q}_{H}$ the set

$$
\{p \in A \mid p(-i) \in H \text { for all } i \geq 1 ; p(j)=e \text { for all } j \geq 0\} .
$$

The proof of Proposition 4.6 will involve several steps and lengthy arguments. We provide a brief outline here for clarity. Following the notation from the statement of Proposition 4.6, we will first show that $[S] \preccurlyeq\left[\left\{B_{+}, t^{ \pm 1}\right\}\right]$. This will allow us to assume, without loss of generality, that $B_{+} \subset Q$. The next step will specify how to identify the required subgroup $H$; it will follow from the definition of $H$ that $\left[S_{H}\right] \preccurlyeq[S]$. The penultimate step will specify how to build all the elements of $\widetilde{Q}_{H}$ in $Q$ by using the conditions of Definition 3.5. The last step will be to use these facts to show that $[S] \preccurlyeq\left[S_{H}\right]$; the equality $[S]=\left[S_{H}\right]$ will obviously follow. We start by introducing some terminology.

Definition 4.7. The negative degree of $p(t) \in R$ is the smallest negative exponent on $t$ that appears in $p(t)$ if it exists. The leading negative coefficient is the coefficient of the term with the negative degree. The degree is the largest positive exponent on $t$ that appears in $p(t)$ if it exists, and the leading coefficient is the coefficient of this term.

For the sake of simplicity, we adopt the convention that our elements from $R$ are written such that terms from left to right have increasing powers on $t$. Thus the leading negative term appears at the extreme left, and the leading term is written on the extreme right.

Example 4.8. The negative degree of $p(t)=2 t^{-9}+3 t^{-6}+1+2 t+t^{6}$ is -9 , and the leading negative coefficient is 2 . The degree is 6 , and leading coefficient is 1 .

The following results will allow us some flexibility in choosing a representative in the equivalence class of $[S]$. This will be very useful in proving Proposition 4.6.

Lemma 4.9. Let $Q \subset R$, and assume that the action of $t$ is strictly confining $R$ into $Q$. Let $f_{j}, j \geq 1$, be a collection of elements from $B_{+} \backslash Q$ that satisfy the following condition: there exists a constant $K \geq 1$ such that $t^{K} f_{j} \in Q$ for all $j \geq 1$. Let $P=\left\{t^{i} f_{j}, \mid j \geq 1, i \geq 0\right\}$ and $\bar{Q}=Q \cup P$. Then the action of $t$ is also strictly confining $R$ into $\bar{Q}$. Further, if $S=\left\{Q, t^{ \pm 1}\right\}$ and $\bar{S}=\left\{\bar{Q}, t^{ \pm 1}\right\}$, then $[S]=[\bar{S}]$.

Proof. We will verify that $\bar{Q}$ satisfies the conditions of Definition 3.5. Observe that $t P \subset P$, and so $t \bar{Q}=t(Q \cup P)=t Q \cup t P \subset Q \cup P=\bar{Q}$. To show the con- 
tainment is strict, observe that, since the elements $f_{j} \in B_{+}$, there exists a $j_{0}$ such that $f_{j_{0}}$ has minimum degree. Then $f_{j_{0}} \in \bar{Q}$, but $f_{j_{0}} \notin t \bar{Q}$. Indeed, if $f_{j_{0}} \in t \bar{Q}$, then either $f_{j_{0}}$ does not have minimum degree or $f_{j_{0}} \in Q$. Both of these are impossible by the hypothesis. This proves condition (a) of Definition 3.5.

Condition (b) of Definition 3.5 is obviously satisfied since $Q \subset \bar{Q}$. It remains to prove condition (c). Let $m_{0}=n_{0}+K$, where $n_{0}$ satisfies condition (c) for $Q$, and $K$ is the constant from the statement of the lemma. It is easy to verify that ${ }^{m_{0}}(\bar{Q}+\bar{Q}) \subseteq Q \subset \bar{Q}$. Thus the first claim holds.

Lastly, we have $\sup _{s \in S}|S|_{S}=1$ since $S \subset \bar{S}$, and by the hypothesis, we have $\sup _{s^{\prime} \in \bar{S}}\left|s^{\prime}\right|_{S} \leq K+1<\infty$. Thus $[S]=[\bar{S}]$.

Corollary 4.10. Let $Q \subset R$, and assume that the action of $t$ is strictly confining $R$ into $Q$. Let $f_{j}, 1 \leq j \leq m$, be a finite collection of elements from $B_{+} \backslash Q$. Let $P=\left\{t^{i} f_{j}, \mid 1 \leq j \leq m, i \geq 0\right\}$ and $\bar{Q}=Q \cup P$. Then the action of $t$ is also strictly confining $R$ into $\bar{Q}$. Further, if $S=\left\{Q, t^{ \pm 1}\right\}$ and $\bar{S}=\left\{\bar{Q}, t^{ \pm 1}\right\}$, then $[S]=[\bar{S}]$.

Proof. For each $j$, there exists a $k_{j} \geq 1$ such that $t^{k_{j}} f_{j} \in Q$ by using condition (b) of Definition 3.5. Take $K=\max \left\{k_{j} \mid 1 \leq j \leq m\right\}$. Then $t^{K} f_{j} \in Q$ for all $1 \leq j \leq m$ by condition (a) of Definition 3.5. The result now follows from Lemma 4.9 with this $K$.

We are now ready to prove the following result, which is the first step in the proof of Proposition 4.6.

Lemma 4.11. Let $Q \subset R$, and let the action of $t$ be confining $R$ into $Q$. Let $S=\left\{Q, t^{ \pm 1}\right\}$. Then $[S] \preccurlyeq\left[\left\{B_{+}, t^{ \pm 1}\right\}\right]$.

Proof. We prove the result by showing that $Q$ can be enlarged to contain all elements from $B_{+}$. First observe that, by Corollary 4.10, we may assume without loss of generality that the constant polynomials $\{0,1, \ldots, n-1\} \subset Q$. Consequently, by using condition (a) of Definition 3.5,

$$
\begin{aligned}
\left\{1, t, t^{2}, \ldots, t^{i}, \ldots\right\} & \cup\left\{2,2 t, 2 t^{2}, \ldots\right\} \\
& \cup \cdots \cup\left\{(n-1),(n-1) t,(n-1) t^{2}, \ldots\right\} \subset Q,
\end{aligned}
$$

i.e., $Q$ contains all elements from $B_{+}$that contain only one term.

For any $0 \leq j<i$ and any $r, s \in \mathbb{Z}_{n}, r t^{j}+s t^{i} \in Q+Q$. By using condition (c) of Definition 3.5, we get that $r t^{n_{0}+j}+s t^{n_{0}+i} \in Q$, i.e., $Q$ contains all elements from $B_{+}$with two terms and such that the smallest exponent on $t$ is $n_{0}$.

Using Lemma 4.9, we may further assume that $Q$ contains all elements from $B_{+}$with exactly two terms. Indeed, this is a consequence of the conclusion of the 
previous paragraph and the observation that every element $p(t) \in B_{+} \backslash Q$ with two terms satisfies $t^{n_{0}} p(t) \in Q$. By iterating the above steps finitely many times, we can conclude that $Q$ can be enlarged to contain all elements from $B_{+}$with at most $n_{0}$ terms.

It now suffices to prove the following claim: for any $n \geq n_{0}, Q$ contains all elements from $B_{+}$with $n$ terms such that the smallest exponent on $t$ is bigger than or equal to $n_{0}$. Indeed, if the claim is proven, then every element $p(t) \in B_{+} \backslash Q$ with more than $n_{0}$ terms will satisfy $t^{n_{0}} p(t) \in Q$. Since $Q$ contains all elements from $B_{+}$with at most $n_{0}$ terms already, it will follow from Lemma 4.9 that $Q$ can be enlarged to contain all elements from $B_{+}$.

To prove the claim, we will use induction on $n \geq n_{0}$. Observe that the base of the induction already holds. Assume that the claim is true for all integers $n$ such that $n_{0} \leq n \leq k$, i.e., $Q$ contains all elements from $B_{+}$with at most $k$ terms such that the smallest exponent on $t$ is $n_{0}$. Let

$$
p(t)=r_{1} t^{n_{0}+i_{1}}+r_{2} t^{n_{0}+i_{2}}+\cdots+r_{n_{0}} t^{i_{n_{0}}+n_{0}}+\cdots+r_{k+1} t^{n_{0}+i_{k+1}},
$$

where $0 \leq i_{1}<i_{2}<\cdots<i_{n_{0}}<\cdots<i_{k+1}$ is a sequence of non-negative integers and $r_{1}, r_{2}, \ldots, r_{k+1}$ is a list of coefficients from $\mathbb{Z}_{n}$.

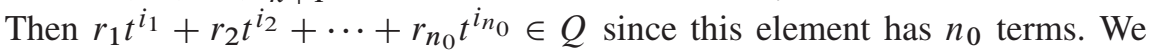
claim that $i_{n_{0}+1} \geq n_{0}$. Indeed, if $i_{n_{0}+1}<n_{0}$, then we cannot choose $n_{0}$ nonnegative integers less than $n_{0}-1$. Thus $r_{n_{0}+1} t^{i_{n_{0}+1}}+\cdots+r_{k+1} t^{i_{k+1}}$ is an element such that the smallest exponent on $t$ is bigger than or equal to $n_{0}$. This element has $k+1-n_{0} \leq k$ terms. If $k+1-n_{0}<n_{0}$, this element is in $Q$. If $k+1-n_{0}>n_{0}$, then this element is in $Q$ by the induction hypothesis.

Thus

$$
r_{1} t^{i_{1}}+r_{2} t^{i_{2}}+\cdots+r_{k+1} t^{i_{k+1}} \in Q+Q .
$$

Using condition (c), we get that $p(t) \in Q$, which completes the proof of the proposition.

We now describe how to identify the subgroup $H<G$ given $Q \subset R$ such that the action of $t$ is confining $R$ into $Q$. Set

$$
\begin{gathered}
H=\langle\text { All coefficients that appear with arbitrarily large negative powers on } t \\
\text { in the elements from } Q\rangle .
\end{gathered}
$$

Example 4.12. Let $G=\mathbb{Z}_{8}$, and $Q$ only contains the elements

$$
4 t^{-i}+2 t^{-i+1}+t^{-1}+b(t) \quad \text { for } i \geq 2, b(t) \in B_{+} .
$$

Then the coefficients that appear with arbitrarily large negative powers of $t$ in the elements of $Q$ are 4 and 2. Thus $H=\langle 4,2\rangle=\{0,2,4,6\}<\mathbb{Z}_{8}$. 
We claim that the subgroup $H$ defined above satisfies $[S]=\left[S_{H}\right]$. To prove this claim, we first establish the following inequality.

Lemma 4.13. $\left[S_{H}\right] \preccurlyeq[S]$.

Proof. For every $k \in \mathbb{Z}_{n}$ which does not appear as one of the generators of $H$, there exists $M_{k} \geq 0$ such that $t^{-M_{k}}$ is the most negative degree with which $k$ appears as a coefficient (we set $M_{k}=0$ if $k$ does not appear as a coefficient of any negative term). Take $M=\max _{k}\left\{M_{k}\right\}$; this is a maximum over a finite set and hence exists. It is easy to verify that $t^{M} q \in Q_{H}$ for every $q \in Q$. Thus $\sup _{q \in Q}|q|_{S_{H}} \leq M+1$, and so $\left[S_{H}\right] \preccurlyeq[S]$.

In order to prove the reverse inequality, we will specify an algorithm that will allow us to build in $Q$ all the elements of $\widetilde{Q}_{H}$. We start by making some observations that will simplify this process.

(1) If $H$ is trivial, then it follows that the elements in $Q$ have a uniformly bounded negative degree. Indeed, there must exist an $M$ such that $t^{M} Q \subset B_{+}$, and so $\left[\left\{B_{+}, t^{ \pm 1}\right\}\right] \preccurlyeq[S]$. From Lemma 4.11, we have $[S]=\left[\left\{B_{+}, t^{ \pm 1}\right\}\right]=\left[S_{\{e\}}\right]$, and so the claim holds in this case. Thus we may assume henceforth that $H$ is non-trivial. Consequently, we may assume that the elements of $Q$ have unbounded negative degrees. In particular, this means that $Q$ has infinitely many polynomials in addition to $B_{+}$.

(2) By Lemma 4.11, we may assume without loss of generality that $B_{+} \subset Q$. Since every element of $R$ has an additive inverse, by adding appropriate elements from $B_{+}$to any element of $Q \backslash B_{+}$and using condition (c) of Definition 3.5, we can reduce to an element of $B_{-}$. In particular, we can assume that the elements we are working with from $Q$ are elements from $B_{-}$when we are in the process of building $\widetilde{Q}_{H}$.

Indeed, the elements of $Q$ given to us may, a priori, not be elements from $B_{-}$ at all, and instead be polynomials with positive and negative powers of $t$. The purpose of this step is to simplify the elements to be from $B_{-}$so that we do not have to worry about the positive powers of $t$; this also makes the notation simpler.

Note that this process does not alter the subgroup $H$ since the coefficients that generate the subgroup occur with arbitrarily small negative powers on $t$. Thus all these coefficients still appear after this step has been executed.

(3) For any element of the form

$$
r_{1} t^{-j_{1}}+r_{2} t^{-j_{2}}+\cdots+r_{k} t^{-j_{k}} \in Q,
$$

where $j_{1}>j_{2}>\cdots>j_{k}>0$, we will refer to the following process as 
recovering an element: using condition (a) of Definition 3.5, multiply the element by $t^{j_{2}}$ to obtain

$$
r_{1} t^{-j_{1}+j_{2}}+b(t) \in Q,
$$

where $b(t) \in B_{+}$. Since $B_{+} \subset Q$, by adding the inverse of $b(t)$ to the above, we get that $r_{1} t^{-j_{1}+j_{2}} \in Q+Q$. Using condition (c) of Definition 3.5, we recover $r_{1} t^{-j_{1}+j_{2}+n_{0}} \in Q$.

We are now ready to describe the required algorithm. Note that we denote the order of an element $g \in \mathbb{Z}_{n}$ by $O(g)$.

Step 1. Since $\mathbb{Z}_{n}$ is finite and the negative degrees of the elements in $Q$ are unbounded, there must exist a coefficient $g_{1} \in \mathbb{Z}_{n}$ and an infinite sequence of elements $\left\{p_{j}\right\}$ of $B_{-} \cap Q$ such that the first term of each $p_{j}$ is of the form $g_{1} t^{-j_{1}}$ and $-j_{1} \rightarrow-\infty$ as $j \rightarrow \infty$.

If infinitely many of the elements $p_{j}$ contain only one term, then we immediately get that $\left\{g_{1} t^{-i} \mid i \geq 1\right\}$ in $Q$ by using condition (a) of Definition 3.5. If not, then there exists a coefficient $g_{2} \in \mathbb{Z}_{n}$ and an infinite subsequence $\left\{q_{j}\right\} \subset\left\{p_{j}\right\}$ such that each $q_{j}$ has the form

$$
g_{1} t^{-j_{1}}+g_{2} t^{-j_{2}}+\cdots .
$$

Such a $g_{2}$ exists because $\mathbb{Z}_{n}$ is finite and the collection $\left\{p_{j}\right\}$ is infinite. Note that $g_{1}$ may equal $g_{2}$ and that $j_{2}$ may be positive or negative.

Step 2. If the difference $j_{1}-j_{2}$ takes arbitrarily large positive values as $j$ varies for the elements $\left\{q_{j}\right\}$, then the values of $-j_{1}+j_{2}+n_{0}$ also take arbitrary large negative values. In this case, by using the process of recovering elements described above and condition (a) of Definition 3.5, we can conclude that

$$
\left\{g_{1} t^{-i} \mid i \geq 0\right\} \in Q \text {. }
$$

Step 3. If the difference $j_{1}-j_{2}$ is bounded for the elements $\left\{q_{j}\right\}$, then we proceed to the following cases. Observe that, in this case, we necessarily have $-j_{2} \rightarrow-\infty$ also. Let $O\left(g_{1}\right)=v$ and $O\left(g_{2}\right)=m$.

Case 3a: $v=m$. Then $g_{1}$ and $g_{2}$ generate the same cyclic subgroup of $\mathbb{Z}_{n}$ so that $\left(g_{1}\right)^{l}=\left(g_{2}\right)^{-1}$ for some $l \geq 1$. Multiply each element $q_{j}$ (which has the form $(*))$ by $t^{j_{1}-j_{2}}$ to obtain the following element in $Q$ by condition (a) of Definition 3.5 since $j_{1}-j_{2}>0$ :

$$
g_{1} t^{-j_{2}}+g_{2} t^{j_{1}-2 j_{2}}+\cdots .
$$

Adding (*) and (\#), we get the following element in $Q+Q$ :

$$
g_{1} t^{-j_{1}}+g_{1} g_{2} t^{-j_{2}}+\cdots \text {. }
$$


By using condition (c) of Definition 3.5, we get

$$
g_{1} t^{-j_{1}+n_{0}}+g_{1} g_{2} t^{-j_{2}+n_{0}}+\cdots \in Q .
$$

By repeating the above steps $l-1$ additional times, we eliminate the second term since $\left(g_{1}\right)^{l} g_{2}=e$, and hence we get new elements of the form

$$
g_{1} t^{j_{1}^{\prime}}+g_{j_{3}}^{\prime} t_{3}^{j_{3}^{\prime}}+\cdots \in Q .
$$

Observe that, since $-j_{1}$ gets arbitrarily negative for all sufficiently large $j$, the exponent $j_{1}^{\prime}$ is also negative for all sufficiently large $j$. Further, the difference between the exponents on $t$ associated to the term with the leading negative coefficient and the immediate next term has been increased, i.e., $\left|j_{1}^{\prime}\right|-\left|j_{3}^{\prime}\right|>j_{1}-j_{2}$. Return to step 1 with the same choice of $g_{1}$ and, if needed, choose an appropriate $g_{2}$ from these new elements.

Case 3b: $v>m$. In this case, we repeatedly use condition (c) $m-1$ times to add each element $q_{j}$ to itself $m-1$ times. By doing so, we eliminate the term $g_{2} t^{-j_{2}}$ and get new elements of the form

$$
\left(g_{1}\right)^{m} t^{j_{1}^{\prime}}+g_{j_{3}}^{\prime} t^{j_{3}^{\prime}}+\cdots \in Q .
$$

Observe that, since $-j_{1}$ gets arbitrarily negative for all sufficiently large $j$, the exponent $j_{1}^{\prime}$ is also negative for all sufficiently large $j$. Further the difference between the exponents on $t$ associated to the term with the leading negative coefficient and the immediate next term has been increased, i.e., $\left|j_{1}^{\prime}\right|-\left|j_{3}^{\prime}\right|>j_{1}-j_{2}$. Return to step 1 with the choice $g_{1}=\left(g_{1}\right)^{m}$ and, if needed, choose an appropriate $g_{2}$ from these new elements.

Case 3c: $v<m$. In this case, we repeatedly use condition (c) $v-1$ times to add each of these elements $(*)$ to itself $v-1$ times. By doing so, we eliminate the leading term $g_{1} t^{-j_{1}}$ and get elements of the form

$$
\left(g_{2}\right)^{v} t^{j_{2}^{\prime}}+g_{j_{3}}^{\prime} t^{j_{3}^{\prime}}+\cdots \in Q .
$$

Observe that, since $-j_{2}$ gets arbitrarily negative for all sufficiently large $j$, the exponent $j_{2}^{\prime}$ is also negative for all sufficiently large $j$. Return to step 1 with the choice $g_{1}=\left(g_{2}\right)^{n}$ and, if needed, choose an appropriate $g_{2}$ from these new elements.

The above process will eventually recover elements of the form $\left\{h t^{-i} \mid i \geq 1\right\}$ in $Q$ for some $h \in H \backslash\{e\}$. We will refer to any element of the form $g t^{i}$, where $g \in \mathbb{Z}_{n}, i \in \mathbb{Z}$ as a monomial, with coefficient $g$ and the set $\left\{g t^{-i} \mid i \geq 1\right\}$ as the complete collection of monomials with coefficient $g$. The above conclusion follows from the following observations: since $\mathbb{Z}_{n}$ is finite, we have finite choices for coefficients. However, since $Q$ contains infinitely many elements with unbounded 
negative degrees, there must be a fixed sequence of coefficients that appear infinitely many times in the elements of $Q$ (possibly with a growing number of terms). The above steps applied to this infinite collection of elements will recover elements with the same coefficients in a specific sequence (which are likely to be different from the original sequence of coefficients).

Since $\left(g_{i}\right)^{n}=1$ for all $i$, we cannot have an infinite sequence of coefficients that decrease in order. Further, $O\left(g^{k}\right)=\frac{O(g)}{\operatorname{gcd}(k, O(g))}$ so that the orders of elements are reducing in step 3 . This means that case $3 \mathrm{c}$ cannot occur infinitely many times in succession, and we eventually have to apply cases $3 \mathrm{a}$ or $3 \mathrm{~b}$. But then the difference between the exponents on $t$ associated to the term with the leading negative coefficient and the immediate next term increase. This allows us to recover elements with the same coefficients, but with larger negative powers on $t$. Consequently, by using condition (a) of Definition 3.5, this process results in recovering the complete collection of monomials corresponding to some $h \in H \backslash\{e\}$.

Step 4. If $O(h)=|H|$, then we are done. Indeed, we can use condition (c) of Definition 3.5 repeatedly to create the complete collection of monomials corresponding to every $h^{\prime} \in H$. To then build $\widetilde{Q}_{H}$ in $Q$, we use induction on the number of terms in elements of $\widetilde{Q}_{H}$ and condition (c) of Definition 3.5.

If $O(h)<|H|$, then we proceed with the following process.

Step 4a. First, we use condition (c) of Definition 3.5 to create all the complete collection of monomials $\left\{h^{j} t^{-i} \mid i \geq 1\right\}$ for every $j \geq 1$. Note that $h$ has finite order. We then use condition (c) again to eliminate all terms that have coefficients from $\langle h\rangle$ from the elements of $Q$. This step, combined with the use of condition (a) of Definition 3.5, might result in the creation of a new complete collection of monomials $\left\{g t^{-i} \mid i \geq 1\right\}$ for some (possibly more than one) $g \in \mathbb{Z}_{n} \backslash\langle h\rangle$. We check to see if $O(g)=|H|$ for some $g \in \mathbb{Z}_{n} \backslash\langle h\rangle$ for which we have a complete collection of monomials. If so, then we are done. If not, we can use condition (c) to create new complete collections of monomials by combining the monomials with coefficients from $\langle h\rangle$ and coefficients from $\mathbb{Z}_{n} \backslash\langle h\rangle$. We again check if any of these new coefficients satisfy $O(g)=|H|$; in which case we are done.

Step $4 \mathrm{~b}$. If step $4 \mathrm{a}$ does not create any complete collection of monomials with a coefficient $g$ satisfying $O(g)=H$, then we let $\widehat{C}$ denote the set that is the union of all the complete collections of monomials we have obtained thus far. We now use condition (c) to eliminate all the terms from $\widehat{C}$ from the elements of $Q$. (Note that this step eliminates all terms with coefficients from $\langle h\rangle$ ). We also use condition (c) to create new coefficients of our choice by combining the terms from elements of $Q$ with the monomials from $\widehat{C}$. We consider the subset $\widehat{Q} \subset Q$ which contains these elements, which have coefficients necessarily distinct from all the coefficients of the monomials of $\widehat{C}$. 
Now we go back to step 1 and repeat the process by choosing the sequence $\left\{p_{j}\right\}$ from $\widehat{Q}$; this ensures that we choose a new $g_{1}, g_{2}$ that are not coefficients of the monomials from $\widehat{C}$ (and hence in $\mathbb{Z}_{n} \backslash\langle h\rangle$ ). This process must in turn also terminate since $|H|<\infty$ and there exists an element $k \in H$ such that $O(k)=|H|$. Thus we get that $\widetilde{Q}_{H} \subset Q$.

Lemma 4.14. $[S] \preccurlyeq\left[S_{H}\right]$. Hence $[S]=\left[S_{H}\right]$.

Proof. Let $q \in Q_{H}$. Then, canonically, $q=\widetilde{q}+b$, where $\widetilde{q} \in B_{-}, b \in B_{+}$. Since $q \in Q_{H}$, we must have $\widetilde{q} \in \widetilde{Q}_{H}$. By the arguments above and Lemma 4.11, we can assume that $\widetilde{Q}_{H}, B_{+} \subset Q$. So we get $q \in Q+Q$. By using condition (c) of Definition 3.5, $t^{n_{0}} q \in Q$. Hence $\sup _{q \in Q_{H}}|q|_{S} \leq n_{0}+1$, which implies that $[S] \preccurlyeq\left[S_{H}\right]$. By Lemma 4.13, $[S]=\left[S_{H}\right]$.

Finally, observe that if $H=G$, then the structure $[S]=\left[S_{H}\right]$ is lineal. Thus if the action of $t$ is strictly confining $R$ into $Q$, then $H$ must be a proper subgroup of $G$. This completes the proof of Proposition 4.6.

We now turn our attention to the lineal structures on $L_{n}$. Our goal is to show that there is a unique lineal structure on $L_{n}$.

Lemma 4.15. Let $[X] \in \mathscr{H}\left(L_{n}\right)$ be any lineal structure. Then $[X]=[A \cup\{t\}]$.

Proof. The subgroup $A \leq L_{n}$ is a characteristic subgroup, and therefore commensurated. Further, $A$ contains no loxodromic elements. It follows from [1, Lemma 4.21] that the induced action of $A$ on $\Gamma\left(L_{n}, X\right)$ must be elliptic. It follows that $[X] \preccurlyeq[A \cup\{t\}]$. By [1, Theorem $4.22(\mathrm{a})], \mathscr{H}_{l}\left(L_{n}\right)$ is always an antichain, and so it follows that $[X]=[A \cup\{t\}]$.

Proof of Theorem 1.4(2). The lamplighter groups have no general type actions. By using the standard ping-pong lemma, one can show that a group which admits a general type action on a hyperbolic space must contain $\mathbb{F}_{2}$, the free group of rank 2, as a subgroup. However, the lamplighter groups are solvable and thus cannot contain non-abelian free subgroups.

By Proposition 4.6, the structures from $\mathbb{S}_{G}$ are the only quasi-parabolic structures on the lamplighter groups. As argued in the proof of Theorem 1.4(1), each of these quasi-parabolic structures dominates a common lineal structure. This lineal structure is unique by Lemma 4.15 . The trivial structure is always unique and dominated by the lineal structure. Thus $\mathcal{B}\left(\mathbb{Z}_{n}\right)=\mathscr{H}\left(\mathbb{Z}_{n}\right.$ wr $\left.\mathbb{Z}\right)=\mathscr{H}\left(L_{n}\right)$.

One might expect that $\mathscr{H}(G$ wr $\mathbb{Z})=\mathscr{B}(G)$ whenever $G$ is finite. However, we have a counter-example to this in the case when $G=\mathbb{Z}_{2} \times \mathbb{Z}_{2}$. The main obstruction in this case is that elements of the same order do not necessarily generate the 
same subgroup (unlike in the case of $\mathbb{Z}_{n}$ ), and this gives rise to quasi-parabolic subgroups that do not correspond to elements of $\mathbb{S}_{G}$. This counterexample also implies that the poset of quasi-parabolic structures on $G$ wr $\mathbb{Z}$ can be very complicated even when $G$ is a very simple group to understand.

Example 4.16. Let $G=\mathbb{Z}_{2} \times \mathbb{Z}_{2}=\{(0,0),(0,1),(1,0),(1,1)\}$. Let $\mathbb{Z}=\langle t\rangle$. For $i \geq 2$, let $p_{i}(t)=(0,1) t^{-i}+(1,0) t^{-i+1}$ be elements in the group ring $R=G[\mathbb{Z}]$ of Laurent polynomials in $t$ over $G$. Set $B_{+}$to be the collection of all elements from $R$ that have terms only with non-negative powers on $t$. Set

$$
Q=\left\{\sum_{k \text { finite }} t^{j_{k}} p_{i_{k}}(t)+b(t) \mid k \geq 0, j_{k} \geq 0, i_{k} \geq 2, b(t) \in B_{+}\right\},
$$

where the notation $\sum_{k \text { finite }}$ means that the sum can range over only a finite collection of choices for $k$, although arbitrarily large.

Clearly, $Q \subset R$. It is understood that, when $k=0$, the sum is an empty sum, and thus we have elements from $B_{+}$. We first show that the action of $t$ is strictly confining $R$ into $Q$. It is clear that $t Q \subseteq Q$. To see the strict containment, consider the element $(1,0) t^{0}=(1,0) \in B_{+} \subset Q$. We will show that $(1,0) t^{-1} \notin Q$, which will imply that $(1,0) \notin t Q$ and hence $t Q \subset Q$. Assume by contradiction that $(1,0) t^{-1} \in Q$. Then we must have

$$
(1,0) t^{-1}=\sum_{k \text { finite }} t^{j_{k}} p_{i_{k}}(t)+b(t)
$$

for some choice of $k, j_{k}$ 's and $p_{i_{k}}$ 's. Observe that $k \neq 0$ since $(1,0) t^{-1} \notin B_{+}$. Also we must have $b(t)=0$ since $(1,0) t^{-1} \in B_{-}$.

Further,

$$
\begin{aligned}
t^{j_{k}} p_{i_{k}}(t) & =t^{j_{k}}\left((0,1) t^{-i_{k}}+(1,0) t^{-i_{k}+1}\right) \\
& =(0,1) t^{-i_{k}+j_{k}}+(1,0) t^{-i_{k}+j_{k}+1} .
\end{aligned}
$$

Fix an $l \geq-1$. If, for any $k,-i_{k}+j_{k}=l$, then the same must hold for an even number of $k$ 's so that these terms cancel amongst themselves in (4.1). Indeed, if this holds for an odd number of $k$ 's, then the sum in (4.1) will have terms from $B_{+}$, which is not possible.

It thus suffices to consider the case when $-i_{k}+j_{k} \leq-2$ for all $k$. In this case, ${ }^{j^{j}} p_{i_{k}}(t)=p_{i_{k}-j_{k}}(t)$ for all $k$. If $i_{k}-j_{k}=i_{k}^{\prime}-j_{k}^{\prime}$ for distinct $k, k^{\prime}$, then these elements will cancel each other since $O(0,1)=O(1,0)=2$. Thus we may assume that the $i_{k}-j_{k}$ 's are all distinct. We may also re-arrange the terms so that if $k$ ranges over $\{1,2, \ldots, n\}$, then $i_{1}-j_{1}>i_{2}-j_{2}>\cdots>i_{n}-j_{n}$. This reduces the sum in (4.1) to

$$
(0,1) t^{-i_{1}+j_{1}}+\cdots+(1,0) t^{-i_{n}+j_{n}+1},
$$


where the terms in the middle have coefficients $(0,1),(1,0)$ or $(1,1)$. But such a sum has at least two non-zero terms and thus cannot equal $(1,0) t^{-1}$.

Since $B_{+} \subset R$, condition (b) of Definition 3.5 holds. It remains to show condition (c). To this end, it is easy to verify that $Q+Q \subseteq Q$. Hence the action of $t$ is strictly confining $R$ into $Q$.

Now let $X=\left\{Q, t^{ \pm 1}\right\}$. We will show that $[X] \neq\left[S_{H}\right]$ and $[X] \neq\left[S_{H}^{\prime}\right]$ for any $H<G$, which will prove the counter-example. Observe that $G=\mathbb{Z}_{2} \times \mathbb{Z}_{2}$ has the following proper subgroups:

$$
\{(0,0)\}, \quad\{(0,0),(0,1)\}, \quad\{(0,0),(1,0)\}, \quad\{(0,0)(1,1)\}
$$

To see that $[X] \neq\left[S_{H}\right]$, we consider the following cases.

Case a: $H=\{(0,0)\}$. Then it is obvious that $\left|p_{i}(t)\right|_{S_{H}} \rightarrow+\infty$ as $i \rightarrow+\infty$.

Case b: $H=\{(0,0),(0,1)\}$. In this case, $Q_{H}$ cannot contain an element where a negative power of $t$ has the coefficient $(1,0)$ since $O(0,1)=2$. Thus we have $\left|p_{i}(t)\right|_{S_{H}} \rightarrow+\infty$ as $i \rightarrow+\infty$. The cases $H=\{(0,0),(1,0)\}$ and $\{(0,0)(1,1)\}$ have similar arguments since $O(1,0)=O(1,1)=2$ also. However, $\left|p_{i}(t)\right|_{X}=1$ for every $i$. Thus $[X] \neq\left[S_{H}\right]$ for any $H<G$.

To see that $[X] \neq\left[S_{H}^{\prime}\right]$ for any $H<G$, recall that $B_{+} \subset Q$. Consider the elements $e_{i}, f_{i}$ for $i \geq 0$ defined by

$$
e_{i}=(0,1) t^{i}, \quad f_{i}=(1,1) t^{i} .
$$

Clearly, since $e_{i}, f_{i} \in B_{+}$for all $i,\left|e_{i}\right|_{X}=1$ and $\left|f_{i}\right|_{X}=1$ for all $i$.

Case a: $H=\{(0,0)\}$ or $H=\{(0,0),(1,0)\}$ or $H=\{(0,0),(1,1)\}$. Then it is easy to see that $\left|e_{i}\right|_{S_{H}^{\prime}} \rightarrow+\infty$ as $i \rightarrow+\infty$.

Case b: $H=\{(0,0),(0,1)\}$. Then $\left|f_{i}\right|_{S_{H}^{\prime}} \rightarrow+\infty$ as $i \rightarrow+\infty$.

Example 4.17 (A group with exactly $\aleph_{0}$ quasi-parabolic structures). Consider the group $U=\bigoplus_{n}\left(\mathbb{Z}_{n}\right.$ wr $\left.\mathbb{Z}\right)$. It follows from [1, Lemma 4.20], that

$$
\mathscr{H}_{\mathrm{gt}}(A \times B)=\mathscr{H}_{\mathrm{gt}}(A) \sqcup \mathscr{H}_{\mathrm{gt}}(B) \quad \text { and } \quad \mathscr{H}_{\mathrm{qp}}(A \times B)=\mathscr{H}_{\mathrm{qp}}(A) \sqcup \mathscr{H}_{\mathrm{qp}}(B)
$$

(where the copies of $\mathscr{H}_{\mathrm{gt}}(A)$ and $\mathscr{H}_{\mathrm{gt}}(B)$ are incomparable in $\mathscr{H}_{\mathrm{gt}}(A \times B)$ and the copies of $\mathscr{H}_{\mathrm{qp}}(A)$ and $\mathscr{H}_{\mathrm{qp}}(B)$ are incomparable in $\left.\mathscr{H}_{\mathrm{qp}}(A \times B)\right)$. Thus we have that $\mathscr{H}_{\mathrm{gt}}(U)$ is empty and $\mathscr{H}_{\mathrm{qp}}(U)=\bigsqcup_{n} \mathscr{H}_{\mathrm{qp}}\left(\mathbb{Z}_{n}\right.$ wr $\left.\mathbb{Z}\right)$. This gives an example of a group with only exactly $\aleph_{0}$ many quasi-parabolic structures.

Example 4.18 (The number of lineal structures may be greater than the number of quasi-parabolic structures on a group). Consider the group $\left(\mathbb{Z}_{2}\right.$ wr $\left.\mathbb{Z}\right) \times \mathbb{Z}$. This group has exactly two quasi-parabolic structures by [1, Lemma 4.20]. However, this group also has uncountably many lineal actions. Indeed, the standard projections to the two copies of $\mathbb{Z}$ in this group yield two linearly independent 
pseudocharacters, and it follows from [1, Theorem 4.22(b)] that there are continuum many lineal structures on this group. This gives an example of a group with a finite number of quasi-parabolic structures, but infinitely many lineal structures. The same argument holds for the groups $\left(\mathbb{Z}_{i}\right.$ wr $\left.\mathbb{Z}\right) \times \mathbb{Z}$ for $i \geq 2$.

Remark 4.19 (Quasi-parabolic structures may not survive under semi-direct products). Let $\mathbb{Z}_{n}=\langle a\rangle$ and $\mathbb{Z}=\langle t\rangle$. Consider the semi-direct product

$$
P_{n}=\left(\mathbb{Z}_{n} \text { wr } \mathbb{Z}\right) \rtimes\langle\alpha\rangle,
$$

where $\alpha \in \operatorname{Aut}\left(\mathbb{Z}_{n}\right.$ wr $\left.\mathbb{Z}\right)$ is the automorphism defined by $\alpha(a)=a, \alpha(t)=t^{-1}$. Then $P_{n}$ has no quasi-parabolic structures. Indeed, it can easily be shown that $t$ must be a loxodromic element in any quasi-parabolic structure of the group $P_{n}$. But then the element $\alpha$ swaps the limit points of $t$, which is impossible in a quasiparabolic action (since one limit point of $t$ must be fixed under the action of the entire group).

Acknowledgments. I am very grateful to P. E. Caprace and Y. de Cornulier for aiding my understanding of [4] by answering my questions. I sincerely thank Gregory Kelsey for helpful conversations about the lamplighter groups. I am also grateful to the anonymous referee for their useful comments during the review process. As always, my heartfelt thanks to my advisor, Denis Osin, for his continued support and advice.

\section{Bibliography}

[1] C. Abbott, S. Balasubramanya and D. Osin, Hyperbolic structures on groups, Algebr. Geom. Topol. 19 (2019), no. 4, 1747-1835.

[2] C. Abbott and A.J. Rasmussen, Actions of solvable Baumslag-Solitar groups on hyperbolic metric spaces, preprint (2019), https : / /arxiv .org/abs/1906.04227.

[3] M. R. Bridson and A. Haefliger, Metric Spaces of Non Positive Curvature, Grundlehren Math. Wiss. 319, Springer, Berlin, 1999.

[4] P.E. Caprace, Y. de Cornulier, N. Monod and R. Tessera, Amenable hyperbolic groups, J. Eur. Math. Soc. (JEMS) 17 (2015), no. 11, 2903-2947.

[5] M. Gromov, Hyperbolic groups, in: Essays in Group Theory, Math. Sci. Res. Inst. Publ. 8, Springer, New York (1987), 75-263.

[6] M. Hamann, Group actions on metric spaces: Ffixed points and free subgroups, preprint (2013), https: / /arxiv .org/abs/1301.6513.

[7] J. F. Manning, Quasi-actions on trees and property (QFA), J. Lond. Math. Soc. (2) 73 (2006), no. 1, 84-108. 
[8] J.F. Manning, Actions of certain arithmetic groups on Gromov hyperbolic spaces, Algebr. Geom. Topol. 8 (2008), no. 3, 1371-1402.

[9] D. Osin, Acylindrically hyperbolic groups, Trans. Amer. Math. Soc. 368 (2016), no. 2, 851-888.

Received September 4, 2018; revised August 18, 2019.

\section{Author information}

Sahana H. Balasubramanya, Department of Mathematics and Statistics, University of North Carolina at Greensboro, Greensboro, NC 27402, USA.

E-mail: hbsahana@gmail.com 